\title{
The eyes see what the mind knows - a need for midgut volvulus ultrasound education: reply to Strouse
}

\author{
HaiThuy N. Nguyen ${ }^{1,2} \cdot$ R. Paul Guillerman ${ }^{1,2} \cdot$ Victor J. Seghers $^{1,2} \cdot$ Marla B. K. Sammer $^{1,2}$ (D) \\ Received: 13 January 2021 / Revised: 13 January 2021 / Accepted: 4 February 2021 / Published online: 19 February 2021 \\ (C) The Author(s), under exclusive licence to Springer-Verlag GmbH, DE part of Springer Nature 2021
}

\section{Dear Editors,}

We appreciate the thoughtful commentary [1] accompanying our recent articles [2,3] on the use of US for midgut volvulus. Dr. Strouse [1] made several vital points. Most significantly, the longer it takes to diagnose midgut volvulus, the greater the risk for bowel necrosis and possible patient death or long-term morbidity due to short gut. Colloquially, time is gut. The case described in the commentary of the 6-day-old with a delayed diagnosis due to lack of recognition on US is particularly salient. Prior to instituting our midgut volvulus US protocol, we, too, had experienced similar cases where a midgut volvulus was depicted on US but not recognized at the time of initial interpretation, leading to a delay in diagnosis made on a subsequent upper gastrointestinal series. The best way for this to be mitigated is for radiologists, radiology trainees and sonographers to be educated, trained and experienced in how to appropriately perform a US exam and recognize the salient sonographic findings for the diagnosis of midgut volvulus on a US that might have been performed for other indications (such as suspected pyloric stenosis, necrotizing enterocolitis or intussusception).

Toward this goal, we advocate for the pediatric radiology community to proactively incorporate the technique into certain routine US exams where practical. For example, assessment of the superior mesenteric vessel alignment, course of the duodenum, and presence or absence of a whirlpool sign could be added to US exams obtained for suspected pyloric stenosis, necrotizing enterocolitis or intussusception, thereby providing cumulative experience and confidence to the sonographers and radiologists in the technique. While midgut volvulus is infrequently encountered, it must be immediately recognized when present on US. Although there is not yet a nationally accepted competency for radiology trainees [4-6], and pediatric

Marla B. K. Sammer

mbsammer@texaschildrens.org

1 Singleton Department of Pediatric Radiology, Texas Children's Hospital, 6701 Fannin St., Houston, TX 77030, USA

2 Department of Radiology, Baylor College of Medicine, Houston, TX, USA sonographer certification guidance does not yet expect competency in midgut volvulus US [7], we implore pediatric radiology departments to add midgut volvulus to their US competencies for their staff, sonographers and trainees. In addition to other resources, we also welcome use of the educational module we use locally and have shared with the radiology community [8].

\section{Declarations}

Conflicts of interest None

\section{References}

1. Strouse PJ (2021) Ultrasound for malrotation and volvulus: has the time come? Pediatr Radiol. https://doi.org/10.1007/s00247-020-04919-3

2. Nguyen HN, Sammer MBK, Ditzler MG et al (2021) Transition to ultrasound as the first-line imaging modality for midgut volvulus: keys to a successful roll-out. Pediatr Radiol. https://doi.org/10. 1007/s00247-020-04913-9

3. Nguyen HN, Navarro OM, Guillerman RP et al (2021) Untwisting the complexity of midgut malrotation and volvulus ultrasound. Pediatr Radiol. https://doi.org/10.1007/s00247-020-04876-x

4. Castro D, Yang J, Greer ML et al (2020) Competency based medical education - towards the development of a standardized pediatric radiology testing module. Acad Radiol 27:1622-1632

5. Mansoori B, Golnari P, Sharifi A et al (2020) Ultrasound training in radiology residency programs: a national survey. J Ultrasound Med. https://doi.org/10.1002/jum.15443

6. Accreditation Council for Graduate Medical Education (2019) Diagnostic radiology milestones. https://www.acgme.org/Portals/0/ PDFs/Milestones/DiagnosticRadiologyMilestones.pdf. Accessed 8 Jan 2021

7. American Registry for Diagnostic Medical Sonography (2014) Job task analysis for ARDMS pediatric sonography. https://www.ardms. org/wp-content/uploads/pdf/PS_Content-Outline-6.20.16.pdf. Accessed 8 Jan 2021

8. Nguyen HN (2021) Midgut volvulus ultrasound. https://sites.google. $\mathrm{com} / \mathrm{view} /$ haithuynguyenmd/home/midgut-volvulus-ultrasound. Accessed 8 Jan 2021

Publisher's note Springer Nature remains neutral with regard to jurisdictional claims in published maps and institutional affiliations. 